\title{
Estado nutricional y actividad física en pacientes pediátricos con diagnóstico oncológico
}

\author{
Nutrition status and physical activity in pediatric patients \\ with cancer diagnosis \\ Estado nutricional e atividade física em pacientes pediátricos \\ com diagnóstico oncológico
}

\author{
Valeria Cruz-Villalba',2, Alda D. García-Guzmán ${ }^{3,4}$, Beatriz A. Pinzón-Navarro', Judith I. Gris-Calvo', \\ Marta Zapata-Tarres ${ }^{3}$, Rocío Cárdenas-Cardos ${ }^{3}$, Isabel Medina-Vera ${ }^{5^{*}}$
}

Recibido: 15 de diciembre 2019. Aceptado para publicación: 3 de marzo 2020.

https://doi.org/10.35454/rncm.v3n1.068

\section{Resumen}

Introducción: la evaluación del estado nutricional y del nivel de actividad física (AF) en pacientes pediátricos diagnosticados con cáncer es de suma importancia debido a que existe una relación entre la mejoría de estas variables y un mejor pronóstico y menores eventos adversos.

Objetivo: evaluar el estado nutricional y el nivel de actividad física en niños con diagnóstico oncológico y la relación con el tipo de diagnóstico y tratamiento oncológico.

Métodos: 83 pacientes (edad 11,3 $\pm 3,4$ años) participaron en este estudio transversal donde se evaluó el estado de nutrición y se determinó su nivel de actividad física a través de los cuestionarios IPAQ-C y IPAQ-A.

Resultados: la prevalencia de desnutrición medida por el Índice de Masa Corporal (IMC) fue 19,2 \%. Sin embargo, al evaluar la reserva muscular a través del perímetro braquial (PB) el porcentaje aumentó a 41,8\%; el puntaje de actividad física, según el Physical Activity Questionnaire fue $1,9 \pm 0,62$ (baja), siendo mayor en el grupo de Leucemias $(2,1 \pm 0,62)$ en comparación con el grupo de Tumores Cerebrales (1,8 $\pm 0,63)$ y Tumores Sólidos $(1,7 \pm 0,63)[p$ $=0,03]$. Aquellos con estado de nutrición normal exhibieron una mayor AF $(2 \pm$ 0,61 ) en comparación con los que tenían

\section{Summary}

Introduction: Assessment of the nutritional status and physical activity level in children with cancer is of the utmost importance as improvement in these factors has been associated with better prognosis and a lower rate of adverse events.

Objective: To assess the nutritional status and physical activity level in children diagnosed with cancer and determine its relationship with type of cancer and treatment given.

Methods: Eighty-three patients (age $11.3 \pm 3.4$ years), participated in this crosssectional study where the nutritional status was assessed, and the level of physical activity was determined using IPAQ-C and IPAQ-A questionnaires.

Results: The prevalence of malnutrition measured by the body mass index was $19.2 \%$. However, when muscle reserves were evaluated using brachial perimeter, the percentage increased to $41.8 \%$; physical activity score according to the Physical Activity Questionnaire was $1.9 \pm$ 0.62 , being higher in the leukemia group $(2.1 \pm 0.62)$ than in the brain and solid tumors groups $(1.8 \pm 0.63$ and $1.7 \pm 0.63$, respectively; $p=0.03$ ). Patients with normal nutritional status showed a significantly greater physical activity compared $(2 \pm$ 0.61) compared with patients with mild

\section{Resumo}

Introdução: a avaliação do estado nutricional e do nível de atividade física (AF) em pacientes pediátricos diagnosticados com câncer é de extrema importância, pois existe uma relação entre a melhoria dessas variáveis e um melhor prognóstico e menores eventos adversos.

Objetivos: avaliar o estado nutricional e o nível de atividade física em crianças com diagnóstico de câncer e a sua relação com o tipo de diagnóstico e tratamento do câncer.

Métodos: 83 pacientes (11,3 $\pm 3,4$ anos) participaram neste estudo transversal em que o estado nutricional foi avaliado e o nível de atividade física foi determinado pelos questionários IPAQ-C e IPAQ-A.

Resultados: a prevalência de desnutrição medida pelo Índice de Massa Corporal foi de $19,2 \%$. No entanto, ao avaliar a reserva muscular através do perímetro braquial $(\mathrm{PB})$, a percentagem aumentou para $41,8 \%$; a pontuação da atividade física, de acordo com o Questionário de Atividade Física, foi de 1,9 $\pm 0,62$ (baixo), sendo maior no grupo Leucemia $(2,1 \pm 0,62)$ em comparação ao grupo Tumores cerebrais $(1,8 \pm 0,63)$ e Tumores sólidos $(1,7 \pm 0,63)$ [ $p=0,03]$. Aqueles com estado nutricional normal exibiram maior FA $(2 \pm 0,61)$ em comparação com aqueles com desnutrição leve $(1,7 \pm$ $0,6)$ e desnutrição moderada $(1,3 \pm 0,37)$ [p 
desnutrición leve $(1,7 \pm 0,6)$ y desnutrición moderada $(1,3 \pm 0,37)[p=0,035]$.

Conclusiones: se observó una alta prevalencia de depleción muscular y desnutrición, el nivel de AF fue bajo (sedentario) sin importar el diagnóstico oncológico. Este conocimiento es de gran relevancia para reafirmar la necesidad del desarrollo de intervenciones dietéticas y de ejercicio adaptadas a las necesidades y habilidades del paciente durante el curso del tratamiento.

Palabras clave: actividad física, cáncer, estado de nutrición, niños. and moderate malnutrition $(1.7 \pm 0.6$ and $1.3 \pm 0.37$ respectively; $p=0.035$ ).

Conclusions: A high prevalence of muscle depletion and malnutrition was observed. In addition, the level of physical activity was low (sedentary) regardless of the type of cancer. This knowledge supports the need to develop dietary and exercise interventions adapted to the needs and capabilities of the cancer patient during the course of treatment.

Keywords: Physical activity; Cancer; Nutritional status; Children.
$=0,035]$.

Conclusões: observou-se alta prevalência de depleção muscular e desnutrição, associada a este baixo nível de FA (sedentário), independentemente do diagnóstico do câncer. Esse conhecimento é de grande relevância para reafirmar a necessidade do desenvolvimento de intervenções alimentares e de exercícios adaptadas às necessidades e habilidades do paciente durante $o$ curso do tratamento.

Palavras-chave: atividade física, câncer, estado nutricional, crianças.

\author{
Servicio de Gastroenterología y Nutrición Pediátrica, Instituto Nacional de \\ Pediatría, Ciudad de México, México. \\ 2 Licenciatura en Nutrición y Ciencia de los Alimentos, Universidad \\ Iberoamericana, Ciudad de México, México. \\ *Correspondencia: Isabel Medina-Vera. \\ imedinav@pediatria.gob.mx
}

\section{INTRODUCCIÓN}

En México, el cáncer es una de las principales causas de muerte infantil, ya que representa la segunda causa de mortalidad $^{(1,2)}$. Además de las consecuencias directas y potencialmente mortales de la enfermedad, el deterioro del estado nutricional se ha relacionado también con tasas altas de mortalidad y complicaciones. La prevalencia de desnutrición al momento del diagnóstico es una variable importante, existe una alta variabilidad que va desde $7 \%$ hasta $50 \%{ }^{(3)}$. El cáncer al inducir un estado catabólico, conduce a cambios en el metabolismo con el aumento de la lipólisis, del gasto energético y de la síntesis de proteínas; esto conduce a una pérdida de masa corporal magra y, en consecuencia, a una pérdida de peso, siendo comunes las pérdidas superiores a $5 \%$ después del primer mes de tratamiento ${ }^{(4)}$. Es por esto que preservar la masa muscular en estos pacientes es importante.

Aunado a lo anterior, los niños también se ven afectados en la calidad de vida y las actividades de la vida diaria. Las actividades físicas habituales en niños $\mathrm{y}$ adolescentes se reducen considerablemente durante y después de las etapas agudas del tratamiento oncológico $^{(5,6)}$; debido al aislamiento al que con frecuencia están propensos en el hospital o en sus casas, lo que causa atrofia muscular y pérdida de la fuerza muscu-

\footnotetext{
3 Departamento de Oncología Médica, Instituto Nacional de Pediatría, Ciudad de México, México.

4 Departamento de Nutrición, Instituto tecnológico y de estudios superiores de Monterrey campus, Ciudad de México, México.

5 Departamento de Metodología de la Investigación, Instituto Nacional de Pediatría, Ciudad de México, México.
}

lar, aumentando el riesgo de fatiga y baja salud ósea y mental ${ }^{(7-9)}$. Estudios previos han demostrado que niños con diagnóstico oncológico presentan deterioro de la condición física y se sabe que esta es multifactorial, uno de los principales contribuyentes además del diagnóstico per se, puede deberse a una disminución de la $\mathrm{AF}^{(8)}$. Por lo tanto, el objetivo del estudio fue evaluar el estado nutricional y el nivel de actividad física $(\mathrm{AF})$ en niños con diagnóstico oncológico y la relación con el tipo de diagnóstico y el tratamiento oncológico.

\section{MÉTODO}

Se llevó a cabo un estudio transversal de marzo a abril de 2019 en el departamento de Oncología Médica del Instituto Nacional de Pediatría, en donde se entrevistó a los pacientes con diagnóstico oncológico en consulta externa, se registraron las variables sexo, edad y diagnóstico oncológico, se cuantificó el nivel de $\mathrm{AF}$, medidas antropométricas y evaluación del estado nutricional como se determina a continuación:

\section{Actividad física}

Se determinó la actividad física (AF) a través de los cuestionarios de auto-reporte: Physical Activity Questionnaire for Older Children (PAQ-C) (si el par- 
ticipante tenía menos de 14 años) y Physical Activity Questionnaire for Adolescents (PAQ-A) (si el participante tenía más de 14 años) los cuales han sido validados previamente ${ }^{(10,11)}$. El nivel de AF se clasificó como bajo, moderado y alto según los puntajes promedio obtenidos de los cuestionarios (1-2,33: bajo, 2,343,66 : moderado y $3,67-5$ : alto ${ }^{(12,13)}$.

\section{Antropometría y estado de nutrición}

La valoración antropométrica incluyó peso $(\mathrm{kg})$, talla $(\mathrm{cm})$, perímetro braquial $(\mathrm{cm})$, área media de brazo $(\mathrm{cm})$. El estado de nutrición fue evaluado por los indicadores Talla para la edad (T/E), Peso para la talla
(P/T), Índice de Masa Corporal (IMC), Perímetro braquial (PB), se clasificaron de acuerdo con los valores establecidos en la NOM-008-SSA2-1993 Control de la nutrición, crecimiento y desarrollo del niño y del adolescente. Criterios y procedimientos para la prestación del servicio ${ }^{(14)}$.

\section{Diagnóstico y tratamiento oncológico}

El diagnóstico oncológico fue emitido por el oncólogo del área médica y fue estratificado en tres grupos: tumores cerebrales, tumores sólidos y leucemias (Tabla 1).

El tratamiento oncológico fue estratificado en tres grupos: en tratamiento, vigilancia/curado y cirugía. El

Tabla 1. Estratificación del diagnóstico oncológico

\begin{tabular}{|c|c|c|}
\hline Tumores cerebrales & Tumores sólidos & Leucemias \\
\hline Astrocitoma pilocítico & Carcinoma nasofaríngeo & Leucemia \\
\hline Ependinoma anaplásico & Ganglioglioma & Leucemia linfoblástica aguda \\
\hline Meduloblastoma & Hepatoblastoma & $\begin{array}{l}\text { Leucemia linfoblástica aguda de alto riesgo } \\
\text { tipo }(+)\end{array}$ \\
\hline Meduloblastoma nodular & Linfomas & Leucemia linfoblástica de células T \\
\hline Neurofibromatosis & Masa mediastinal & Leucemia linfoblástica tipo B \\
\hline \multirow[t]{17}{*}{ Tumor frontal } & Osteocitoma & Leucemia mieloide aguda \\
\hline & Osteosarcomas & \\
\hline & Rabdomiosarcoma & \\
\hline & Retinoblastoma & \\
\hline & Ruiz Maldonado con Virus de Epstein Barr & \\
\hline & Sarcoma de Ewing & \\
\hline & Síndrome proliferativo & \\
\hline & Teratoma sacrococcígeo & \\
\hline & Tumor de senos endodérmicos en el ovario & \\
\hline & Tumor de Wilms & \\
\hline & Tumor en el oído & \\
\hline & Tumor en la tiroides & \\
\hline & Tumor en ovario & \\
\hline & Tumor maligno de huesos largos & \\
\hline & Tumor maligno nasofaríngeo & \\
\hline & Tumor miofibroblástico recidivante & \\
\hline & Tumor rabdoide extrarrenal & \\
\hline
\end{tabular}


grupo de tratamiento involucró a aquellos pacientes que se encontraban recibiendo quimioterapia ambulatoria y radioterapia, con enfermedad activa y aquellos que se encontraban en remisión (pacientes que estaban recibiendo quimioterapia ambulatoria, quimioterapia en hospitalización y radioterapia), sin enfermedad activa; en el grupo de vigilancia/curado se incluyeron aquellos pacientes que terminaron el tratamiento oncológico y solo se encontraban en visitas periódicas al servicio de oncología para su evaluación; los curados se tomaron en cuenta como pacientes que llevaban más de 2 años en vigilancia; en el grupo de cirugía se anexaron pacientes que a pesar de estar recibiendo quimioterapia y radioterapia, en el momento de la entrevista, se encontraba saliendo de algún tipo de procedimiento quirúrgico, ya sea resección tumoral o amputación de algún miembro.

\section{Análisis estadístico}

Para el análisis descriptivo, las variables cuantitativas fueron expresadas como promedio \pm desviación estándar y las variables cualitativas como porcentaje. Para comparar las diferencias de los puntajes de AF entre los tipos de diagnóstico oncológico, de tratamiento y el estado de nutrición se utilizó la prueba ANOVA de una vía, con análisis post-hoc LSD (mínima diferencia significativa, por su sigla en inglés). El valor significativo de p se estableció en $<0,05$. Los datos fueron analizados por medio del programa SPSS (versión 25, SPSS Inc, Chicago, IL y GraphPad Prism 7).

\section{Consideraciones éticas}

El estudio sigue los principios éticos de la Declaración de Helsinki y adhiere la Ley General de Salud de los Estados Unidos Mexicanos en materia de Investigación para la Salud. Se conceptualiza como una investigación sin riesgo debido a que solo se recolectan datos relacionados con cada uno de los sujetos sin ponerlos en situación de riesgo. Este estudio es parte del protocolo aprobado (número 059/2019) por los Comités de Ética e Investigación del Instituto Nacional de Pediatría, registrados oficialmente en la Oficina de Protección de la Investigación Humana del NIH (http://ohrp.cit.nih.gov/search/search.aspx) con los números IRB00008064 e IRB00008065.

\section{RESULTADOS}

Se estudiaron en total 83 pacientes pediátricos, $54,2 \%$ corresponde al sexo femenino, la edad promedio fue de
$11,3 \pm 3,4$ años teniendo una edad mínima de 6 años y máxima de 17,8 años. Los diagnósticos con mayor prevalencia fueron tumores sólidos $(54,2 \%)$, seguidos de leucemias $(33,8 \%)$ y posteriormente tumores cerebrales (12\%) (Tabla 2).

Tabla 2. Características generales de los participantes

\begin{tabular}{|c|c|}
\hline & $\mathrm{n}=\mathbf{8 3}(\%)$ \\
\hline \multicolumn{2}{|l|}{ Diagnóstico } \\
\hline Tumores cerebrales & $10(12)$ \\
\hline Tumores sólidos & $45(54,2)$ \\
\hline Leucemias & $28(33,8)$ \\
\hline \multicolumn{2}{|l|}{ Etapa de tratamiento, n (\%) } \\
\hline Tratamiento & $59(71,1)$ \\
\hline Vigilancia / Curado & $20(24,1)$ \\
\hline Cirugía & $3(3,6)$ \\
\hline Remisión & $1(1,2)$ \\
\hline \multicolumn{2}{|l|}{ Antropometría } \\
\hline Peso & $39,3 \pm 14$ \\
\hline Talla & $141 \pm 18$ \\
\hline Talla / Edad, puntaje Z & $-0,71 \pm 1,13$ \\
\hline Talla / Edad, \% & $96,5 \pm 5,03$ \\
\hline Peso / Talla, puntaje Z & $0,2 \pm 2,7$ \\
\hline Peso / Talla, \% & $114 \pm 32$ \\
\hline Perímetro braquial, cm & $21,4 \pm 3,7$ \\
\hline Perímetro braquial, puntaje Z & $-0,96 \pm 1,22$ \\
\hline Índice de Masa Corporal, kg/m² & $19 \pm 3,9$ \\
\hline Índice de Masa Corporal, puntaje Z & $0,04 \pm 1,7$ \\
\hline Área Media de Brazo, cm & $23,5 \pm 9$ \\
\hline Área Media de Brazo, \% & $91,6 \pm 28,2$ \\
\hline \multicolumn{2}{|c|}{$\begin{array}{l}\text { Tratamiento: quimioterapia, radioterapia y radioterapia con } \\
\text { quimioterapia. }\end{array}$} \\
\hline
\end{tabular}

Al evaluar el estado nutricional de los pacientes pediátricos a través de puntaje $\mathrm{Z}$ para IMC se observó, de manera global, que 51,8 \% tenía un índice normal, al analizarlo por estrato de diagnóstico oncológico las leucemias presentaron un porcentaje mayor de niños en el rango de normalidad (60,7 \%), seguido de tumores cerebrales $(50 \%)$ y de tumores sólidos $(46,7 \%)$; sin embargo, de manera global se presentó $28,9 \%$ de sobre- 
peso/obesidad y 19,2 \% de algún grado de desnutrición (Figura 1A); de manera interesante pudimos observar que cuando evaluamos el estado de nutrición por medio del PB que es un indicador de masa muscular, el porcentaje de desnutrición se eleva de una forma alarmante $(41,8 \%)$ y el de sobrepeso/obesidad disminuye a 3,8 \% (Figura 1B). Los resultados del análisis de la AF indicaron que de manera global el promedio del puntaje fue 1,9 $\pm 0,62$ determinándolo como una $\mathrm{AF}$ baja. $\mathrm{Al}$ realizar el análisis por estrato de diagnóstico oncológico, el grupo de diagnóstico de leucemias obtuvo un puntaje promedio mayor de $\operatorname{AF}(2,1 \pm 0,62)$ que, aunque fue mayor en comparación al grupo de tumores cerebrales $(1,8 \pm 0,63)$ y tumores sólidos $(1,7 \pm 0,63)[p=0,03]$, todos ellos se catalogan como AF leve (Figura 2A). Sin embargo, al categorizar de manera individual el nivel de AF solo $27,2 \%$ de la población en general está en el rango de AF moderada. 40,7 \% de los individuos con diagnóstico de leucemia, 33,3\% de los individuos con diagnóstico de tumores cerebrales y solo $17,8 \%$ de los individuos con tumores sólidos obtuvieron una puntuación para $\mathrm{AF}$ moderada (Figura 2B).

$\mathrm{Al}$ analizar el nivel de AF relacionado con el estado de nutrición y a la etapa de tratamiento; para el primero observamos que de acuerdo con el indicador Talla/ Edad (porcentaje) los que tuvieron un estado normal

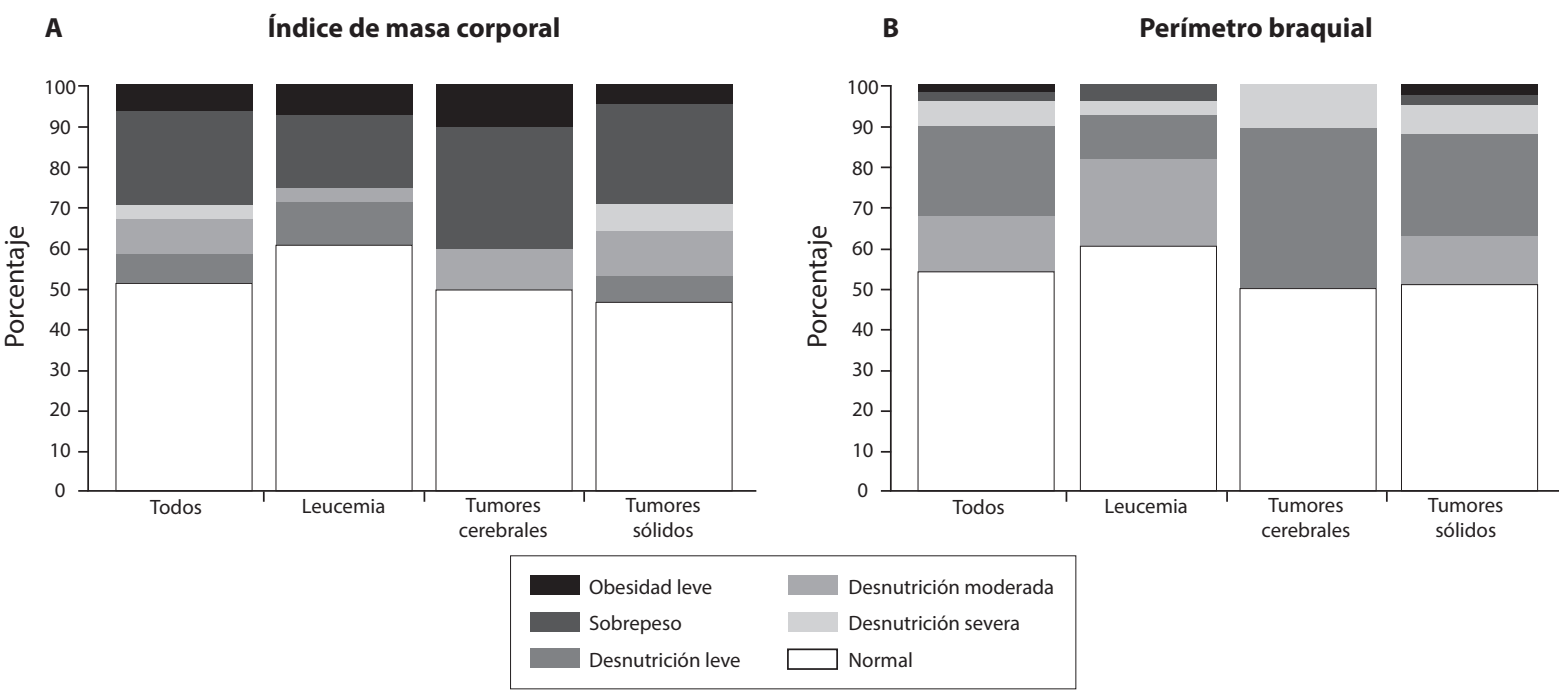

Figura 1. Evaluación del estado nutricional por diagnóstico oncológico. A. Estado de nutrición por indicador Índice de Masa Corporal (puntaje Z). B. Estado de nutrición a través del perímetro braquial (puntaje Z).

A

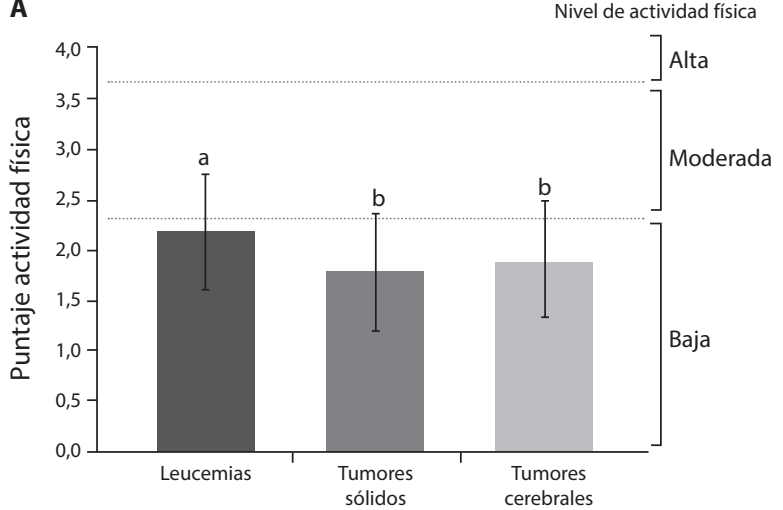

B

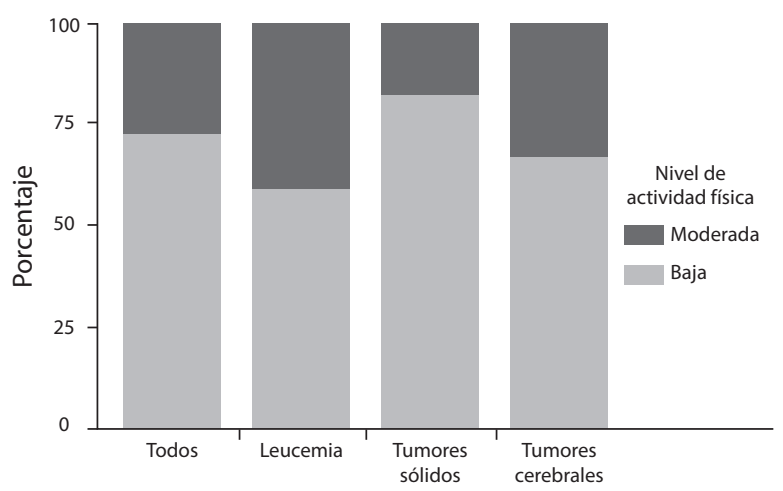

Figura 2. Actividad física en niños por diagnóstico oncológico. A. Puntaje de actividad física estratificado por grupo de diagnóstico oncológico. Barras que no presentan la misma letra son significativamente diferentes (valor $-p<0,05)$. B. Porcentaje de niños con actividad física moderada o baja. 
exhibieron una mayor AF $(2 \pm 0,61)$ en comparación con los que tenían desnutrición leve $(1,7 \pm 0,6)$ y desnutrición moderada $(1,3 \pm 0,37)[\mathrm{p}=0,035]$ (Figura $3 \mathrm{~A})$. De acuerdo con la etapa de tratamiento en aquellos que estaban en vigilancia/curados observamos una AF más alta $(2,1 \pm 0,58)$ en comparación con aquellos que estaban en tratamiento con quimioterapia, radioterapia o una combinación de ambos $(1,8 \pm 0,61)$ y aquellos que estaban en cirugía $(1,3 \pm 0,39)[\mathrm{p}=0,033]$ (Figura 3B).

\section{DISCUSIÓN}

El objetivo del presente estudio fue evaluar el estado nutricional en población pediátrica con diagnóstico oncológico, una prevalencia medida por el indicador de puntaje $\mathrm{Z}$ para IMC de 19,2\% de desnutrición, siendo mayor la prevalencia en el estrato de aquellos que tuvieron diagnóstico de tumor sólido $(24,4 \%)$, estrato en el que se ha reportado con mayor riesgo de empeorar la desnutrición ${ }^{(15)}$.

$\mathrm{Al}$ analizar la desnutrición según el $\mathrm{PB}$ encontramos una prevalencia mayor de desnutrición (40.8\%), lo que revela una importante depleción muscular, con implicaciones trascendentes debido a que a pesar de que el paciente podría encontrarse en el peso ideal o incluso en sobrepeso/obesidad la depleción muscular es evidente. Es importante recalcar que, aunque no existe un estándar de referencia universalmente aceptado para el diagnóstico de desnutrición, el PB es un fuerte indicador de cantidad de masa muscular ${ }^{(16)}$ reconocido por la Organización Mundial de la Salud. Es necesario un acuerdo internacional para estandarizar la definición y evaluación de la desnutrición de tal manera que permita comparar de forma adecuada estas prevalencia en población pediátrica.

También parte de nuestro estudio se enfocó en medir el nivel de AF en niños con diagnóstico oncológico y evaluar la relación de esta actividad de acuerdo con el tratamiento oncológico y el estado de nutrición. Los resultados mostraron un nivel promedio general de $\mathrm{AF}$ bajo, no obstante, encontramos un nivel mayor de AF en aquellos niños con diagnóstico de Leucemia, pero aún así siendo clasificado como bajo; resultados similares han sido reportados por Winter, et al. ${ }^{(5)}$, donde se encontró que los pacientes con tumor de hueso y leucemia diferían tanto en la cantidad como en la intensidad de la actividad física siendo los pacientes con tumores óseos menos activos. Por otro lado, Yee Tan, et al. ${ }^{(17)}$ demostraron que los pacientes pediátricos sometidos a tratamiento para la leucemia fueron significativamente menos activos que los niños controles sanos. Si bien, al ser estratificada el nivel de AF encontramos que 27,2\% de nuestra población alcanzó un nivel de AF moderada. Las recomendaciones en pacientes oncológicos es evitar la inactividad física durante y después del tratamiento. El panel de expertos del American College of Sports Medicine (ACSM) concluyó sobre las pautas para el ejercicio en adultos sobrevivientes de cáncer que existe una amplia evidencia de que el ejercicio es seguro tanto durante como después del tratamiento para todos los tipos de cáncer, las recomendaciones generales van desde 30 a 60 minutos de AF moderada a intensa, al menos 5 días a la semana, pero teniendo en cuenta las consideraciones especiales necesarias para garantizar la seguridad de esta población potencialmente vulnerable
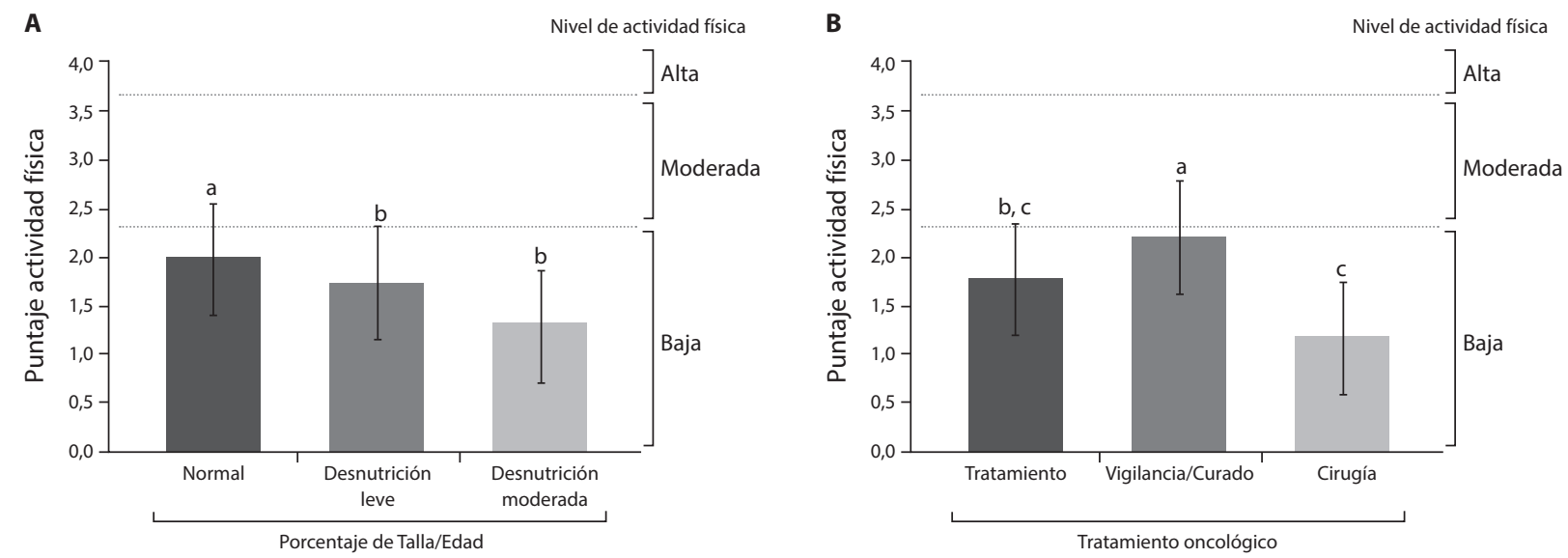

Figura 3. Actividad física por estado de nutrición y etapa del tratamiento. A. Puntaje de actividad física por estrato de estado de nutrición. B. Puntaje de actividad física por etapa de tratamiento oncológico. Barras que no presentan la misma letra son significativamente diferentes (valor $-p<0,05$ ). 
y evaluar las condiciones inherentes como fatiga y náuseas, entre otras ${ }^{(18)}$. Por lo tanto, alentar a los pacientes pediátricos, por medio de intervenciones de $\mathrm{AF}$, ya sea en el ambiente hospitalario, en el comunitario o en sus casas, a alcanzar estas metas podrían tener efectos benéficos en esta población, ya que se ha reportado que la $\mathrm{AF}$ durante el tratamiento oncológico no solo contribuye al desarrollo motor y salud física, psicológica y social, sino que también protege contra la recurrencia y mortalidad del cáncer ${ }^{(19)}$.

La AF estratificada por tratamiento oncológico mostró que aquellos pacientes en vigilancia/curados tuvieron una AF significativamente más alta en comparación con aquellos que se encontraban recibiendo quimioterapia, radioterapia o ambos tratamientos, y con aquellos que se encontraban en etapa peri operatoria, esta diferencia de AF entre tratamientos puede deberse a la falta de movilidad de los pacientes que han sido sometidos a una cirugía reciente, y por otro lado, los pacientes que se encuentran recibiendo quimioterapia o radioterapia pueden llegar a presentar fatiga $u$ otro tipo de molestias después de recibir el tratamiento que no les permite llegar al nivel de AF que realizan los pacientes que se encuentran en vigilancia/curados ${ }^{(20)}$.

\section{Limitaciones del estudio}

Dentro de las limitaciones del presente estudio se encuentra el uso de cuestionarios de reporte para el nivel de AF. Sin embargo, estos cuestionarios han sido validados para cuantificar el nivel de AF. Además, en el estudio se utilizó la medición antropométrica de $\mathrm{PB}$ para evaluar la depleción muscular, debido a que es una medición práctica, no invasiva y de bajo costo, la cual ha demostrado ser un buen indicador de la masa muscular ${ }^{(21,22)}$. Sin embargo, no hay acuerdo en los valores de corte de PB y pueden ser específicos para edad y población; por lo tanto, medir la composición corporal por medio de otros métodos como absorciometría de rayos $\mathrm{X}$ de energía dual (DEXA), bioimpedancia eléctrica, etc. podría dar un valor más certero de masa muscular, aunque este tipo de evaluaciones son más costosas y requieren mayor tiempo en la medición.

\section{CONCLUSIÓN}

La desnutrición es una condición altamente prevalente en población pediátrica con diagnóstico de cáncer, aunque la inactividad física en esta población es un problema a menudo observado no es cuantificado con frecuencia, la actividad física en pacientes con cáncer se reduce considerablemente, y los pacientes con tumores sólidos tienen mayor prevalencia de desnutrición y menor nivel de actividad física, por lo tanto, mayor riesgo de los efectos colaterales de la afección del estado nutricional y de los efectos perjudiciales de la inactividad prolongada. Tanto nuestros hallazgos en este estudio como el hecho aceptado de que la actividad es un elemento esencial del desarrollo infantil confirman la necesidad del desarrollo de intervenciones dietéticas y de ejercicio adaptadas a las necesidades y habilidades del paciente durante el curso del tratamiento.

\section{Declaración de autoría}

MV I, GG AD, PN BA, fueron responsables de la concepción y diseño del estudio, CV V, GG AD, GC JI, realizaron el estudio y participaron en la adquisición y recopilación de datos. ZT M, CC R, realizaron las evaluaciones relacionadas a diagnósticos oncológicos, GG AD, PN BA, MVI, interpretación de los datos, MV I, realizó el análisis estadístico y la redacción del artículo; todos los autores participaron en la revisión crítica de los datos y del manuscrito y tuvieron acceso total a todos los datos del estudio y compartieron la responsabilidad de la decisión final de presentar este informe para su publicación.

\section{Conflicto de intereses}

No existe conflicto de interés por parte de los autores.

\section{Financiamiento}

No se recibió financiamiento de ningún tipo.

\section{Referencias bibliográficas}

1. Rivera-Luna R, Zapata-Tarres M, Shalkow-Klincovstein J, Velasco-Hidalgo L, Olaya-Vargas A, Finkelstein-Mizrahi N, et al. The burden of childhood cancer in Mexico: Implications for low- and middle-income countries. Pediatr Blood Cancer. 2017; 64(6). Doi: 10.1002/pbc.26366

2. Rivera-Luna R, Correa-Gonzalez C, Altamirano-Alvarez E, Sánchez-Zubieta F, Cárdenas-Cardós R, Escamilla-Asian G, et al. Incidence of childhood cancer among Mexican children registered under a public medical insurance program. Int J Cancer. 2013;132 (7):1646-50. Doi:10.1002/ijc.27771

3. Selwood K, Ward E, Gibson F. Assessment and management of nutritional challenges in children's cancer care: a survey of current practice in the United Kingdom. Eur J Oncol Nurs. 2010;14(5): 439-46. Doi: 10.1016/j.ejon.2010.04.004.

4. Rickard KA, Grosfeld JL, Kirksey A, Ballantine TV, Baehner RL. Reversal of protein-energy malnutrition in children 
during treatment of advanced neoplastic disease. Ann Surg. 1979;190(6):771-81. Doi:10.1097/00000658-19791200000018

5. Winter C, Müller C, Brandes M, Brinkmann A, Hoffmann C, Hardes J, et al. Level of activity in children undergoing cancer treatment. Pediatr Blood Cancer. 2009;53(3):438-43. Doi: $10.1002 / \mathrm{pbc} .22055$.

6. Winter CC, Müller C, Hardes J, Boos J, Gosheger G, Rosenbaum D. Pediatric patients with a malignant bone tumor: when does functional assessment make sense? Support Care Cancer. 2012;20(1):127-33. Doi: 10.1007/ s00520-010-1077-5.

7. Braam KI, van Dijk EM, Veening MA, Bierings MB, Merks JH, Grootenhuis MA, et al. Design of the Quality of Life in Motion (QLIM) study: A randomized controlled trial to evaluate the effectiveness and cost-effectiveness of a combined physical exercise and psychosocial training program to improve physical fitness in children with cancer. BMC Cancer. 2010;10:624. Doi: 10.1186/1471-2407-10-624.

8. Thorsteinsson T, Helms AS, Adamsen L, Andersen LB, Andersen KV, Christensen KB, et al. Study protocol: Rehabilitation including Social and Physical activity and Education in Children and Teenagers with cancer (RESPECT), BMC Cancer. 2013;13: 544.Doi: 10.1186/1471-2407-13-544.

9. Götte M, Caroline-Seidel C, Verena-Kesting S, Rosenbaum $\mathrm{D}$, Boos J. Objectively measured versus self-reported physical activity in children and adolescents with cancer. PLOS One. 2017;12(2): e0172216. Doi: 10.1371/journal.pone.0172216.

10. Janz KF, Lutuchy EM, Wenthe P, Levy SM. Measuring activity in children and adolescents using self-report: PAQ-C and PAQ-A. Med Sci Sport Exer. 2008;40(4):767-72. Doi: 10.1249/MSS.0b013e3181620ed1.

11. Herazo-Beltrán AY, Domínguez-Anaya R. Confiabilidad del cuestionario de actividad física en niños colombianos. Rev Salud Pública. 2012; 14(5):802-9.

12. Dan-SP Jr, Mohd-Nasir MT, Zalilah MS. Sex and ethnic differentials in physical activity levels of adolescents in kuantan. Malays J Nutr. 2007;13(2):109-20.

13. Wan Dali WPE, Jan Mohamed HJ, Yusoff H. Nutrient Intakes Status and Physical Inactivity among Overweight and Obese School Children in Kota Bharu, Kelantan, Malaysia. Iran J Public Health. 2018;47(8):1098-107.33. PMID: 30186781.
14. Comité Consultivo Nacional de Normalización de Servicios de Salud, Secretaría de Salud, Estados Unidos Mexicanos. Norma Oficial Mexicana NOM-008-SSA2-1993, Control de la nutrición, crecimiento y desarrollo del niño y del adolescente. Criterios y procedimientos para la prestación del servicio [Internet]. México D.F.: 28 de julio de 1994. [Fecha de consulta: 13 de mayo 2019] Disponible en: http://www. salud.gob.mx/unidades/cdi/nom/008ssa23.html

15. Gaynor EP, Sullivan PB. Nutritional status and nutritional management in children with cancer. Arch Dis Child. 2015;100(12):1169-72. Doi: 10.1136/archdischild-2014-306941

16. Antillon F, Rossi E, Molina AL, Sala A, Pencharz P, Valsecchi MG, et al. Nutritional Status of Children During Treatment for Acute Lymphoblastic Leukemia in Guatemala. Pediatr Blood Cancer. 2013;60(6):911-5. Doi: 10.1002/pbc.24377.

17. Tan SY, Poh BK, Chong HX, Ismail MN, Rahman J, Zarina AL, et al. Physical activity of pediatric patients with acute leukemia undergoing induction or consolidation chemotherapy. Leuk Res. 2013; 37(1):14-20. Doi: 10.1016/j.leukres.2012.09.005.

18. Williams L, Wilkins L. ACSM's guidelines for exercise testing and prescription. 9th ed. Baltimore (MD): Wolters Kluwer; 2014.

19. Cormie P, Zopf EM, Zhang X, Schmitz KH. The impact of exercise on cancer mortality, recurrence, and treatment-related adverse effects. Epidemiol Rev. 2017; 39(1):71-92. Doi: 10.1093/epirev/mxx007.

20. Astruc E. Physical Activity Guidelines for Children During and After Cancer Treatment [Master's thesis]. The College at Brockport; 2016. [Fecha de consulta: 13 de mayo 2019] Disponible en: http://digitalcommons.brockport.edu/honors/145.

21. Chomtho S, Fewtrell MS, Jaffe A, Williams JE, Wells JC. Evaluation of arm anthropometry for assessing pediatric body composition: evidence from healthy and sick children. Pediatr Res. 2006; 59(6):860-5. Doi: 10.1203/01. pdr.0000219395.83159.91.

22. Jordão AA, Bellucci AD, Dutra de Oliveira JE, Sérgio Marchini J. Mid arm computerized tomography fat, muscle and total areas correlation with nutritional assessment data. Int J Obes Relat Metab Disord. 2004;28(11):1451-5. Doi: 10.1038/ sj.ijo.0802781. 